\title{
A histochemical comparison of the O-acylated sialic acids of the epithelial mucins in ulcerative colitis, Crohn's disease, and normal controls
}

\author{
C. F. A. CULling, P. E. REID, AND W. L. DUNN \\ From the Department of Pathology, Faculty of Medicine, University of British Columbia, \\ Vancouver, BC, Canada V6T 1 W5
}

SUMmary Two histochemical techniques, the PAT/KOH/PAS and the PBT/KOH/PAS, were used to investigate the side chain $\mathrm{O}$-acyl substitution patterns of the sialic acids of the colonic epithelial mucins in cases of ulcerative colitis and Crohn's disease. In both diseases there was, as compared to normal, a reduction in the proportion of sialic acids $\mathrm{O}$-acylated at $\mathrm{C}_{7} \mathrm{C}_{8}$, the reduction being greater in ulcerative colitis. Further, there appeared to be an association between the severity of the disease and the reduction in the staining of $\mathrm{O}$-acylated sialic acids. This relationship was more marked in ulcerative colitis. In some cases of both diseases there was evidence for epithelial mucins containing predominantly $\mathrm{C}_{7}$-substituted sialic acids.

This study has confirmed our previous conclusion that, in Crohn's disease of the terminal ileum, the disease is associated with an increase in the proportion of sialic acids bearing side chain substituents.

In previous histochemical and chemical investigations we have described methods for the identification of the side chain and/or $\mathrm{C}_{4} \mathrm{O}$-acylated sialic acids which are present as terminal, non-reducing, residues on the epithelial glycoproteins of the large intestine and terminal ileum of man (Culling et al., $1971 ; 1974 \mathrm{a} ; 1974 \mathrm{~b} ; 1975 ; 1976 ; 1977 \mathrm{a} ; 1977 \mathrm{~b}$; Reid et al., 1973; 1974; 1975a; 1975b; 1976; 1977; $1978 \mathrm{a} ; 1978 \mathrm{~b})$. The presence of these side chain O-acylated sialic acids has proved to be useful in the identification of metastases arising from carcinomas of the colon (Culling et al., 1975), in the diagnosis of perianal Paget's disease (Wood and Culling, 1975), and for the differentiation of carcinomas arising in anal gland epithelium and rectal mucosa (Fenger and Filipe, 1977). Changes in the side chain substitution pattern of these acids were found to be associated with malignancy in the colon (Culling et al., 1977a) and Crohn's disease of the terminal ileum (Culling et al., 1977b).

In this communication we report the study of a series of specimens from cases of ulcerative colitis and Crohn's disease of the large intestine.

Received for publication 8 May 1979

\section{Material and methods}

\section{TISSUES}

Specimens from cases diagnosed as ulcerative colitis or Crohn's disease were obtained from the surgical pathology department of the Vancouver General Hospital. They were either biopsies, iliectomy or colectomy specimens and were all formol calcium fixed and paraplast embedded. The following tissues were examined: 28 specimens of large intestine from 13 cases of ulcerative colitis; 42 specimens of large intestine and 50 specimens of ileum from 17 cases of Crohn's disease; within the latter group, the disease was confined to the small intestine in three cases and to the large intestine in four others. Sections of normal colon and ileum were used as controls. All sections were stained by haematoxylin and eosin and graded for severity of the disease on a scale from 1 to 5. The criteria used to assess the severity of the disease in individual sections from cases of ulcerative colitis were:

Grade 1 No disease present.

Grade 2 Showing early signs of the disease, eg, crypt abscesses, some cellular infiltration, and eosinophilia, but no ulceration.

Grade 3 Superficial erosion of mucosal surface, marked cellular infiltration, increased vascularity, 
and even early destruction of epithelial tubules. Grade 4 Marked ulceration with a polypoid surface and intense vascularity, destruction of epithelial tubules, and some thinning of muscle layers.

Grade 5 Acute fulminating disease with extensive ulceration presenting little or no crypt architecture, intense vascularity, and marked thinning of muscle layers.

The criteria used to assess the severity of the disease present in individual sections from cases of Crohn's disease were:

Grade 1 No disease present.

Grade 2 Section shows early signs of disease, eg, superficial ulceration, aphthoid ulcers, some enlargement of lymphoid follicles, and Peyer's patches.

Grade 3 Marked ulceration with early fissuring, lymphoid hyperplasia, oedema, and transmural inflammatory changes.

Grade 4 Marked fissuring with transmural lymphoid hyperplasia, sarcoid type granulomas, and some fibrous tissue formation.

Grade 5 Acute fulminating disease with marked thickening of submucosa by oedema, fibrosis, and inflammatory cell infiltrate. Usually sarcoid type granulomas and focal arteritis are seen.

\section{HISTOCHEMICAL PROCEDURES}

(a) Haematoxylin and eosin to demonstrate microanatomy (Culling, 1974)

(b) Periodic acid/sodium borohydride/saponification/ PAS technique (PBT/KOH/PAS) (Reid et al., 1973)

Tissue vicinal diols were oxidised with periodic acid, and the engendered aldehydes were reduced with sodium borohydride to PAS negative, primary alcohols. Subsequent saponification removed O-acyl ester substituents from potential vicinal diols which rendered them PAS positive. Positive staining by this technique has been shown to be associated with sialic acids bearing side chain substituents at positions $\mathrm{C}_{7}$ or $\mathrm{C}_{8}$ or which are di- or tri-substituted (Culling et al., 1974a).

Sections were graded from 0 to 4 based upon the intensity of red staining of the epithelial mucins (the only material stained).

(c) Periodic acid-thionin Schiff/saponification/PAS technique (PAT/KOH/PAS) (Culling et al., 1976)

Reactive vicinal diols were oxidised with periodic acid and then stained blue with a thionin Schiff reagent. After saponification those vicinal diols produced as a result of alkali treatment were demonstrated by the conventional PAS technique.

Using this procedure, all normally PAS reactive sites were stained blue while sialic acids substituted at position $\mathrm{C}_{8}$ (or which are di- or tri-substituted) were stained red. Based upon the shade of staining of the mucins, sections were classified as either blue (0), blue-purple (1), purple (2), red-purple (3), or red (4) and assigned the numbers in parentheses to determine averages.

A good staining control can be produced by staining a colectomy specimen of normal colon by PBT/KOH/PAS techniques. This will show the intense red assigned a grade of 4 with some mucin present being grade 3 ; the PAT/KOH/PAS on the same sections will demonstrate mucin stained either red or red-purple; the staining intensities reflect the degree of O-acylation of the sialic acids present in the epithelial mucins. The relative validity of the visual assessments made upon slides stained by these two techniques has been supported by both correlative chemical analysis (Reid et al., 1978b) and microspectrophotometric measurements (Culling et al., 1979, unpublished observations).

\section{Results}

The results obtained from the studies of ulcerative colitis and Crohn's disease of the colon and ileum are presented in Tables 1 and 2 respectively. It should be noted that in those cases where more than one block was examined the results are presented as an average reading from all the blocks. It will be seen that, although there were some variations in staining between cases, the staining patterns we obtained were reasonably consistent for both ulcerative colitis and Crohn's disease. The differences between normal and diseased bowel were made more obvious by the consistent staining of the normal colon, which was invariably within the range of 3-4 by the PBT/KOH/PAS procedure and red to red-purple by the PAT/KOH/PAS techniques.

Table 1 Average readings of the degree of severity and staining reactions of cases of ulcerative colitis

\begin{tabular}{rllll}
\hline Case & $\begin{array}{l}\text { No. of } \\
\text { blocks }\end{array}$ & $\begin{array}{l}\text { Degree of } \\
\text { severity }\end{array}$ & $\begin{array}{l}\text { PAT/KOH/PAS } \\
\text { colour }\end{array}$ & $\begin{array}{l}\text { PBT/KOH/PAS } \\
\text { reaction* }\end{array}$ \\
\hline 1 & 6 & 3 & BP & $1 \frac{1}{2}$ \\
2 & 1 & $3-4$ & BP & $1 \frac{1}{2}$ \\
3 & 1 & 3 & BP-P & 2 \\
4 & 1 & $2-3$ & BP & $3+$ \\
5 & 1 & 2 & P & $3+$ \\
6 & 6 & 4 & BP & $1 \frac{1}{2}$ \\
7 & 1 & 4 & B-BP & 2 \\
8 & 1 & 3 & BP & 2 \\
9 & 5 & $3 \frac{1}{2}$ & P-BP & 2 \\
10 & 1 & 3 & B-BP & 2 \\
11 & 1 & 3 & B & $1 \frac{1}{2}$ \\
12 & 2 & $2-3$ & P-RP & 3 \\
13 & 1 & $3-4$ & B & 2 \\
\hline
\end{tabular}

$\mathbf{B}=$ blue; $\mathbf{P}=$ purple

$* 4=$ strong; $3=$ moderate; 2 = weak; $1=$ very weak 
Table 2 Average readings of the degree of severity and staining reactions of cases of Crohn's disease of large intestine

\begin{tabular}{rllll}
\hline Case & $\begin{array}{l}\text { No. of } \\
\text { blocks }\end{array}$ & $\begin{array}{l}\text { Degree of } \\
\text { severity }\end{array}$ & $\begin{array}{l}\text { PAT/KOH/PAS } \\
\text { colour }\end{array}$ & $\begin{array}{l}\text { PBT/KOH/PAS } \\
\text { reaction* }\end{array}$ \\
\hline 1 & 3 & $2-3$ & R-RP & $3 \frac{1}{2}$ \\
2 & 7 & 4 & BP & 3 \\
4 & 3 & $3 \frac{1}{2}$ & BP & $2 \frac{1}{2}$ \\
5 & 1 & 2 & P & 3 \\
6 & 1 & $2-3$ & RP & $2 \frac{1}{2}$ \\
7 & 2 & $3 \frac{1}{2}$ & BP & $2 \frac{1}{2}$ \\
8 & 2 & 3 & P-BP & $2 \frac{1}{2}$ \\
9 & 2 & $2 \frac{1}{2}$ & B-BP & $2 \frac{1}{2}$ \\
10 & 3 & 4 & BP & $1 \frac{1}{2}$ \\
11 & 1 & 2 & BP-RP & $2 \frac{1}{2}$ \\
12 & 7 & 4 & P & $2 \frac{1}{2}$ \\
14 & 5 & 4 & P-BP & $1 \frac{1}{2}$ \\
15 & 4 & 4 & P & 2 \\
17 & 1 & 3 & BP & $2 \frac{1}{2}$ \\
\hline
\end{tabular}

$\mathbf{R}=$ red; $\mathbf{B}=$ blue; $\mathbf{P}=$ purple

*4 = strong; $3=$ moderate; $2=$ weak; 1 = very weak

\section{ULCERATIVE COLITIS}

There was a reduced amount of mucin present in these specimens, particularly in the more severely ulcerated areas. All the specimens showed a marked reduction in those sialic acids $\mathrm{O}$-acylated at $\mathrm{C}_{8}$ (in which we include $\mathrm{C}_{7,8} ; \mathrm{C}_{7,9} ; \mathrm{C}_{8,9}$ or $\mathrm{C}_{7,8,9}$ ) (see Table 1), which appeared to be associated with the grade of severity of the disease, as defined by the criteria above. While the 15 sections graded 2-3 for severity exhibited a wide range of staining, in general, they showed consistently less staining for $\mathrm{O}$-acylated sialic acids than normal. In contrast, 10 sections from the more severely diseased specimens (graded 3.5-5) showed a narrower range, and a greater loss of staining, for O-acylated sialic acids.

\section{CROHN'S DISEASE}

\section{Colon}

These specimens (Table 2) showed more overall variation in staining than the sections of ulcerative colitis. There was a reduction in side chain O-acetylation as compared to normal, but this reduction was not as marked as in ulcerative colitis.

These changes in staining appeared to be associated with the severity of the disease. The 17 sections graded between 2 and 3 had a less marked reduction in O-acylated sialic acid than those $\mathbf{2 5}$ sections from more severely diseased specimens (graded 3.5-5). The relative differences were not, however, as marked as those seen in ulcerative colitis.

\section{Ileum}

Our results showed that, as compared to mucin from normal ileum, there was an increase in staining for sialic acids bearing side chain substituents. These results confirm our previous observations (Culling et al., 1977b).

C7 O-ACYLATED SIALOMUCINS

Sialic acids $\mathrm{O}$-acylated at $\mathrm{C}_{7}$ were considered to predominate in those mucins which stained blue by the PAT/KOH/PAS and red by the PBT/KOH/PAS (Culling et al., 1976). For the identification of such mucins artificial limits were set, which we believe to be conservative, which included those mucins that were blue by PAT/KOH/PAS and 1.5 (or more) by $\mathrm{PBT} / \mathrm{KOH} / \mathrm{PAS}$, or were blue-purple and 2.5 (or more) respectively. Such defined mucins occurred in three of 13 cases of ulcerative colitis, in four of 14 cases of Crohn's disease in large intestine, and in four of 13 cases of Crohn's disease of small intestine.

\section{Discussion}

Histochemical investigations of the epithelial mucins of the large intestine of man have shown that they are composed predominantly of acid non sulphated and acid sulphated mucosubstances, sialo- and sulphomucins respectively, and that the distribution of the latter varies with both the level of the crypt and the anatomic region of the colon (Lev and Spicer, 1965; Greco et al., 1967; Hellstrom and Fisher, 1967; Hradsky et al., 1967; Goldman and Ming, 1968; Filipe, 1969; 1971; 1972; Gad, 1969a; 1969b; Johansen and Kay, 1969; Filipe and Dawson, 1970; Korhonen et al., 1971; Mäkelä et al., 1971; Subbuswamy, 1971a; 1971b; Dawson, 1972; Filipe and Branfoot, 1974; 1976; Lev and Orlic, 1974). We have demonstrated that the sialic acids in such mucins bear $\mathrm{O}$-acyl substituents at position $\mathrm{C}_{4}$ and/or at various positions on the polyhydroxy side chain. In man, such side chain substituted sialic acids are apparently evenly distributed along the length of the crypt and the length of the colon (Culling et al., 1971; Reid et al., 1973). Chemical studies of normal human colonic epithelial glycoproteins (Gold and Miller, 1974; Reid et al., 1975a) have demonstrated the presence of a molecular specie containing both sialic acid and $\mathrm{O}$-sulphate ester.

There is no general agreement as to the nature of the changes in the epithelial mucins that occur in ulcerative colitis and Crohn's disease. Hellstrom and Fisher (1967) concluded that ulcerative colitis could be distinguished from Crohn's disease because in the former there was a severe decrease in mucin. Filipe (1969) and Filipe and Dawson (1970) found a similar sharp discrimination in rectal biopsies. In the opinion of Filipe and Branfoot (1976), ... .So long as one ignores areas of severe ulceration and polypoidal hyperplasia (where mucins are respect- 
ively absent or increased), the mucins are qualitatively and quantitatively normal even in the presence of inflammation in Crohn's colitis, but in ulcerative colitis they are reduced proportionately with the severity of the inflammation . . $\therefore$ The general reduction in mucin affected the sulphomucins to a greater extent than the sialomucins while neutral mucins were decreased or absent (Filipe, 1969; Filipe and Dawson, 1970; Dawson, 1972). In contrast, Greco et al. (1967) found that in areas of ulcerative colitis showing only 'slight histological change' the neutral mucosubstances were not confined to the lower two-thirds of the crypts but were distributed along the whole crypt, moreover they appeared in the sigmoid where they were practically absent in normals. They found there was an increase in intracellular acid mucin in superficial weakly acid goblet cells and noted an absolute increase in sulphomucin. In areas of 'severe histological change' they found a significant decrease and sometimes even a total absence of neutral mucin with apparently only weakly acidic mucosubstances persisting.

The results of the present study suggested that in ulcerative colitis, as compared to normal, there was a reduction in the overall quantity of mucin, particularly in the more severely ulcerated areas, which confirms the findings of others. There was also a marked reduction in the proportion of sialic acids O-acylated at $\mathrm{C}_{7,8}$, which appeared to be associated with the severity of the disease. In Crohn's disease there was also a reduction, from normal, in side chain $\mathrm{O}$-acylation of the sialic acids in the mucin, and some degree of association between these changes and the severity of the disease. However, in Crohn's disease, these differences from normal were not as marked as those in ulcerative colitis. While there was therefore undoubtedly a pattern of change between these diseases and normal and between the diseases themselves, the difference was not sufficiently clearcut to be of great diagnostic value, since the actual diversity of results made individual cases difficult to evaluate. In spite of this, however, we do feel that such staining provides another dimension which could be of some assistance in making a differential diagnosis.

In a previous study (Culling et al., 1977b), a loss of staining for O-acylated sialic acids appeared to be an indicator of malignant change in the colon, and it is interesting that the incidence of malignancy is high in ulcerative colitis and, as has been shown recently, is increased in Crohn's disease (Huizenga, 1978).

Filipe and Branfoot (1976) have reported changes in the epithelial mucins in long-standing colitis similar to those described for cancer in the general population (Filipe, 1969; 1972; Filipe and Branfoot,
1976). It is tempting, therefore, to suggest that there is a general mechanism whereby loss or impairment of the mechanism for the O-acylation of sialic acids can lead to ulcerative colitis and perhaps malignancy. Such a hypothesis could possibly explain the association of cancer with ulcerative colitis and Crohn's disease.

Approximately $25 \%$ of the sections from both Crohn's disease and ulcerative colitis, and $30 \%$ of those from small intestine with Crohn's disease, contained mucin that was predominantly $\mathrm{O}$-acylated at $\mathrm{C}_{7}$. We previously encountered this type of mucin in our study of colonic malignancy (Culling et al., 1977b). We are currently engaged in an in-depth study of adenocarcinoma of colon, during which it is hoped that the significance of these findings will become apparent.

We have suggested (Culling et al., 1974a) that the $\mathrm{O}$-acylated sialic acids in the colonic mucins are part of a protective mechanism against the actions of the faecal stream. Accepting such a hypothesis, our results suggest that a pivotal event in ulcerative colitis, and possibly Crohn's disease, might be an interference with the synthesis of $\mathrm{O}$-acylated sialic acids, rendering the mucins, and consequently the underlying mucosa, more vulnerable to attack by the faecal stream. This suggestion is made more attractive by the observations of Van Der Waaij et al. (1974), who found that carrageenan-induced ulcerative disease of the large intestine of guinea-pigs was significantly 'mitigated' by selective elimination of certain bacteria.

We have reported previously (Culling et al., 1977a) that Crohn's disease of the terminal ileum is accompanied by an increase in the proportion of side chain $\mathrm{O}$-acylated sialic acids in the mucin, and the present study has confirmed this finding. This increase might be an attempt by the small intestine to provide protection against some other unknown factor or alternatively indicates a different pathogenesis for ulcerative colitis as compared to Crohn's disease.

This work was supported by the Medical Research Council of Canada.

It is a pleasure to acknowledge the superior technical skill of Mrs Odile Polo and Mrs Jana Tomanek and the secretarial assistance of Miss Sandra Sturgeon.

\section{References}

Culling, C. F. A. (1974). Handbook of Histopathological and Histochemical Techniques, 3rd edition. Butterworth, London.

Culling, C. F. A., Reid, P. E., Burton, J. D., and Dunn, 
W. L. (1975), A histochemical method of differentiating lower gastrointestinal tract mucin from other mucins in primary or metastatic tumours. Journal of Clinical Pathology, 28, 656-658.

Culling, C. F. A., Reid, P. E., Clay, M. G., and Dunn, W. L. (1974a). The histochemical demonstration of O-acylated sialic acid in gastrointestinal mucins: their association with the potassium hydroxide-periodic acid-Schiff effect. Journal of Histochemistry and Cytochemistry, 22, 826-831.

Culling, C. F. A., Reid, P. E., and Dunn, W. L. (1971). The effect of saponification upon certain histochemical reactions of the epithelial mucins of the GI tract. Journal of Histochemistry and Cytochemistry, 19, 654-662.

Culling, C. F. A., Reid, P. E., and Dunn, W. L. (1974b). $A$ problem in the removal of sialic acid by acid hydrolysis. Stain Technology, 49, 317-318.

Culling, C. F. A., Reid, P. E., and Dunn, W. L. (1976). A new histochemical method for the identification and visualisation of both side chain acylated and nonacylated sialic acids. Journal of Histochemistry and Cytochemistry, 24, 1225-1230.

Culling, C. F. A., Reid, P. E., and Dunn, W. L. (1977a). Histochemical comparison of the epithelial mucins in the ileum in Crohn's disease and in normal controls. Journal of Clinical Pathology, 30, 1063-1067.

Culling, C. F. A., Reid, P. E., Worth, A. J., and Dunn, W. L. (1977b). A new histochemical technique of use in the interpretation and diagnosis of adenocarcinoma and villous lesions in the large intestine. Journal of Clinical Pathology, 30, 1056-1062.

Dawson, I. M. P. (1972). The histochemistry of Crohn's disease. Clinics in Gastroenterology, 1, 309-320.

Fenger, C., and Filipe, M. I. (1977). Pathology of the anal glands with special reference to their mucin histochemistry. Acta Pathologica et Microbiologica Scandinavica, 85A, 273-285.

Filipe, M. I. (1969). Value of histochemical reactions for mucosubstances in the diagnosis of certain pathological conditions of the colon and rectum. Gut, 10, 577-586.

Filipe, M. I. (1971). ${ }^{35}$ Sulphur uptake in the mucosa adjacent to carcinoma of the large intestine. Histochemical Journal, 3, 27-35.

Filipe, M. I. (1972). The value of a study of the mucosubstances in rectal biopsies from patients with carcinoma of the rectum and lower sigmoid in the diagnosis of premalignant mucosa. Journal of Clinical Pathology, 25, 123-128.

Filipe, M. I., and Branfoot, A. C. (1974). Abnormal patterns of mucus secretion in apparently normal mucosa of large intestine with carcinoma. Cancer, 34, 282-290.

Filipe, M. I., and Branfoot, A. C. (1976). Mucin histochemistry of the colon. Current Topics in Pathology, 63, 143-178.

Filipe, M. I., and Dawson, I. M. P. (1970). The diagnostic value of mucosubstances in rectal biopsies from patients with ulcerative colitis and Crohn's disease. Gut, 11, 229-234.

Gad, A. (1969a). A histochemical study of human alimentary tract mucosubstances in health and disease.
I. Normal and tumours. British Journal of Cancer, 23, 52-63.

Gad, A. (1969b). A histochemical study of human alimentary tract mucosubstances in health and disease. II. Inflammatory conditions. British Journal of Cancer, 23, 64-68.

Gold. D. V., and Miller, F. (1974). Characterization of human colonic mucoprotein antigen. Immunochemistry, 11, 369-375.

Goldman, H., and Ming, S. C. (1968). Mucins in normal and neoplastic gastrointestinal epithelium: histochemical distribution. Archives of Pathology, 85, 580-586.

Greco, V., Lauro, G., Fabbrini, A., and Torsoli, A. (1967). Histochemistry of the colonic epithelial mucins in normal subjects and in patients with ulcerative colitis. Gut, 8, 491-496.

Hellstrom, H. R., and Fisher, E. R. (1967). Estimation of mucosal mucin as an aid in the differentiation of Crohn's disease of the colon and chronic ulcerative colitis. American Journal of Clinical Pathology, 48, 259-268.

Hradsky, M., Langr, R., Nerad, V., and Skaunic, V. (1967). Alterations of the histological and histochemical pattern in ulcerative colitis biopsy specimens. American Journal of Proctology, 18, 392-398.

Huizenga, K. A. (1978). Colonic cancer in inflammatory bowel disease. Mayo Clinic Proceedings, 53, 474-475.

Johansen, P. G., and Kay, R. (1969). Histochemistry of rectal mucus in cystic fibrosis of the pancreas. Journal of Pathology, 99, 299-306.

Korhonen, L. K., Mäkelä, V., and Lilius, G. (1971). Carbohydrate-rich compounds in the colonic mucosa of man. II. Histochemical characteristics of colonic adenocarcinomas. Cancer, 27, 128-133.

Lev, R., and Orlic, D. (1974). Histochemical and radioautographic studies of normal human fetal colon. Histochemistry, 39, 301-311.

Lev, R., and Spicer, S. S. (1965). A histochemical comparison of human epithelial mucins in normal and in hypersecretory states including pancreatic cystic fibrosis. American Journal of Pathology, 46, 23-47.

Mäkelä, V., Korhonen, L. K., and Lilius, G. (1971). Carbohydrate rich compounds in the colonic mucosa of man. I. Histochemical characteristics of normal and adenomatous colonic mucosa. Cancer, 27, 120-127.

Reid, P. E., Culling, C. F. A., and Dunn, W. L. (1973). Saponification-induced increase in the periodic acidSchiff reaction in the gastrointestinal tract: mechanism and distribution of the reactive substance. Journal of Histochemistry and Cytochemistry, 21, 473-482.

Reid, P. E., Culling, C. F. A., and Dunn, W. L. (1974). The histochemical interpretation of the complex results of methylation upon gastrointestinal tract mucins, with special reference to the periodic acid-Schiff reactivity. Journal of Histochemistry and Cytochemistry, 22, 986-991.

Reid, P. E., Culling, C. F. A., and Dunn, W. L. (1978a). A histochemical method for the identification of 9-0acyl sialic acids : an investigation of bovine submaxillary gland and intestinal mucin. Journal of Histochemistry and Cytochemistry, 26, 187-192. 
Reid, P. E., Culling, C. F. A., Dunn, W. L., and Clay, M. G. (1976). The use of a transesterification technique to distinguish between certain neuraminidase resistant epithelial resistant epithelial mucins. Histochemistry, 46, 203-207.

Reid, P. E., Culling, C. F. A., Dunn, W. L., Clay, M. G., and Ramey, C. W. (1978b). A correlative chemical and histochemical study of the O-acetylated sialic acids of human colonic epithelial glycoproteins in formalin fixed paraffin embedded tissues. Journal of Histochemistry and Cytochemistry, 26, 1033-1041.

Reid, P. E., Culling, C. F. A., Dunn, W. L., Ramey, C. W., and Clay, M. G. (1975b). Differences in chemical composition between the epithelial glycoproteins of the upper and lower halves of rat colon. Canadian Journal of Biochemistry, 53, 1328-1332.

Reid, P. E., Culling, C. F. A., Ramey, C. W., Dunn, W. L., and Clay, M. G. (1977). A simple method for the determination of the O-acetyl substitution pattern of the sialic acids of colonic epithelial glycoprotein. Canadian Journal of Biochemistry, 55, 493-503.

Reid, P. E., Culling, C. F. A., Tsang Wai-Chui, Clay, M. G., Ramey, C. W., and Dunn, W. L. (1975a). The demonstration of $\mathrm{O}$-acetylated sialic acids in colonic epithelial glycoproteins. Canadian Journal of Biochemistry, 53, 388-391.

Subbuswamy, S. G. (1971a). Patterns of mucin secretion in human intestinal mucosa. Journal of Anatomy, 108, 291-293.

Subbuswamy, S. G. (1971b). Mucosubstances in neoplasms of the human colon and rectum. Gut, 12, 200-207.

Van Der Waaij, D., Cohen, B. J., and Anver, M. R. (1974). Mitigation of experimental inflammatory bowel disease in guinea pigs by selective elimination of the aerobic gram negative intestinal microflora. Gastroenterology, 67, 460-472.

Wood, W. S., and Culling, C. F. A. (1975). Perianal Paget disease: histochemical differentiation utilising the borohydride-KOH-PAS reaction. Archives of Pathology, 99, 442-445.

Requests for reprints to: Professor C. F. A. Culling, Department of Pathology, The University of British Columbia, 2075 Wesbrook Mall, Vancouver, BC, Canada V6T 1W5. 